\title{
Identification of the Unmet Social Needs of Community- dweller Elderly with Cognitive Impairment Using WHOQOL-OLD Form
}

Perdamaian T. ${ }^{1}$, Widagdo T.M.M., Sigilipoe M.A., Manus W.C.,

Centre for Research and Service on Gerontology

${ }^{1}$ Faculty of Medicine, Universitas Kristen Duta Wacana, Yogyakarta, Indonesia,

Background: Frailty is marked by declining cognitive function and social interaction. Palliative approach in frail elderly should include identification of unmet social needs which might help caregivers and social workers to optimise patient's quality of life (QoL).

Aim: This study is aimed to identify unmet social needs in elderly with cognitive impairment using WHOQoL-OLD form.

Methods: A cross-sectional study was conducted in 14 sub-districts of Yogyakarta City, Indonesia, among 377 community dweller seniors ( $\geq 60$ years old) in 2017. Cognitive impairment is defined as adjusted-MMSE score $\leq 24$. The Indonesian version of WHOQoL-OLD form was administered with researcher assistance. We conducted multilevel modelling with maximum likelihood approach ( $n / m e$ ver. 3.1-137 in R) to predict whether cognitive impairment would show poorer QoL profile, with sub-districts as random variable. The limit of significance in this study is 0.05 .
Results: There were 80 (21.22\%; $95 \% \mathrm{Cl}=17.27$ $25.77 \%$ ) elderly people classified with cognitive impairment. The average $\mathrm{QoL}$ is $83.84 \pm 7.90 \%$ in healthier group, and $80.52 \pm 9.57 \%$ in impaired cognitive group. The presence of cognitive impairment significantly predict overall $\mathrm{CoL}(b=$ $0,24, t(362)=-2.47, p=0,014)$, Autonomy domain $(b=0,05, t(362)=-2.82, p=0.005)$, and Social Participation domain $(b=-0,04, t(362)=-2,98, p=$ $0,003)$ with negative correlation $(r=-0,224$, 0,228 , and $r=-0,357$, subsequently). Within impaired cognitive group, MMSE score predict the Sensory domain $(b=-0.01, t(65)=-1,99, p=$ $0,050)$ with negative correlation $(r=-0,983)$.

Discussion: Elderly with cognitive impairment showed poorer QoL, especially in Autonomy and Social domain, compared to healthier group. In addition, lower MMSE score is associated with poor sensory abilities. This study demonstrated that WHO-QoL OLD form is useful to identify the social needs, independency and sensory decline in elderly which indicate early social support.

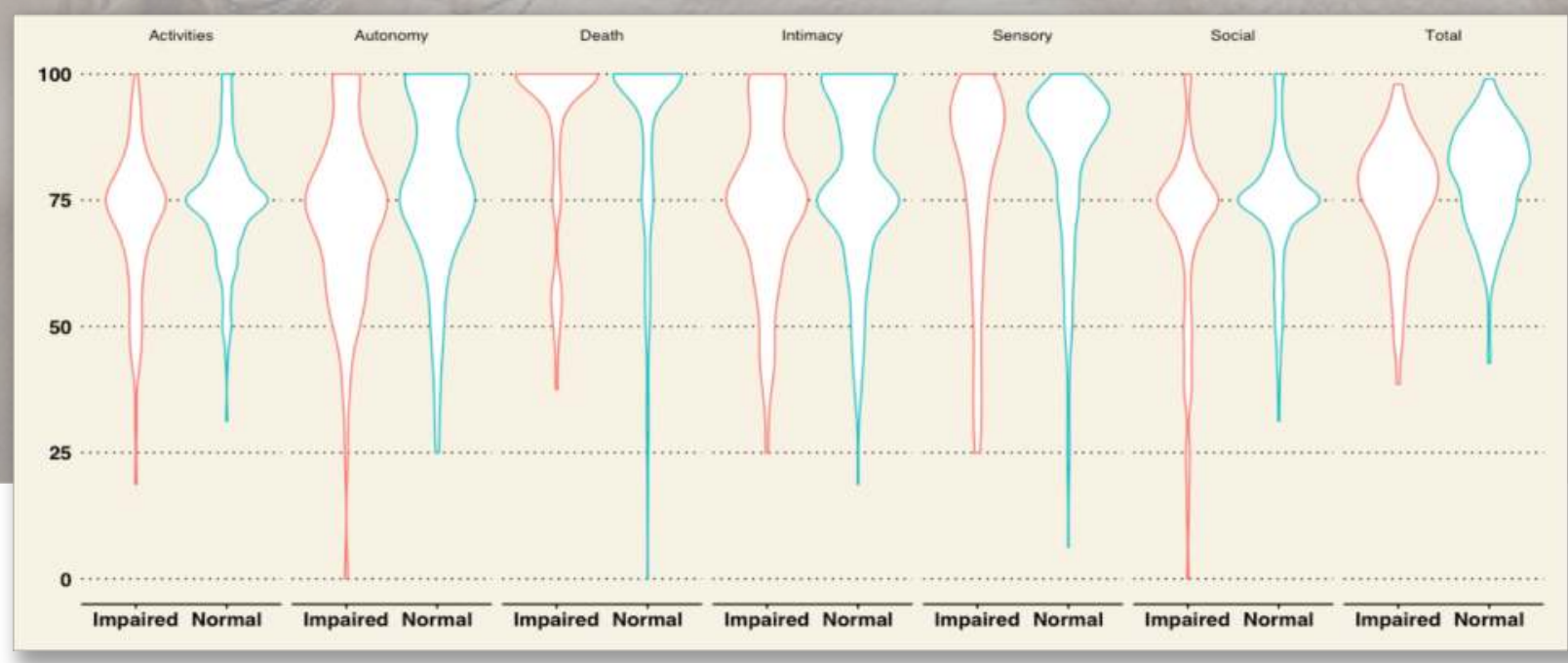

Fig 1. Distribution of QoL Score for in each domain according respondent's cognitive status (Impaired $(n=80)$ vs normal $(n=297)$ ) 